Classification

Physics Abstracts

74.50

\title{
Caractérisation magnétique d'un bilame supraconducteur *
}

\author{
J. Chilo \\ Laboratoire d'Electromagnétisme, U.A. 833, ENSERG, 23, rue des Martyrs, 38031 Grenoble Cedex, France
}

(Reçu le 5 avril 1985, révisé le 27 juin 1985, accepté le 2 juillet 1985)

\begin{abstract}
Résumé. - Par un développement analytique direct, nous caractérisons complètement les propriétés magnétiques d'un bilame supraconducteur. Nous en déduisons sa profondeur de pénétration équivalente ainsi que son inductance globale. En fixant le courant total traversant ce bilame, nous donnons également les distributions de densité de courant dans une section droite du dispositif. Les résultats analytiques présentés sont en parfait accord avec ceux obtenus par des méthodes de résolution numérique ou par des tests interférométriques.
\end{abstract}

\begin{abstract}
We propose a straightforward analysis for magnetic characterization of a double layered strip. We take out the equivalent London penetration depth and the resulting inductance. The total current supported by such a line being fixed, we determine the distributions of the current density. Close agreement with experimental and numerical results confirm the validity of the proposed method.
\end{abstract}

\section{Introduction.}

L'intérêt des jonctions Josephson utilisant des électrodes en $\mathrm{NbN}$ réside dans leurs tensions gap importantes ( $4 \mathrm{mV}$ environ) et leurs bonnes tenues lors des recyclages thermiques [1]. Cependant la grande profondeur de London $\lambda$ de ce matériau (typiquement $4000 \AA$ ) est à l'origine d'une inductance cinétique qui augmente rapidement dès que l'épaisseur du $\mathrm{NbN}$ devient inférieure à $\lambda$ [2]. Il en résulte une diminution de la sensibilité des dispositifs interférométriques [3] et un accroissement du temps de propagation dans les dispositifs logiques [4].

Certains auteurs ont proposé de remplacer l'électrode de base de la jonction Josephson par un bilame $\mathrm{Nb}-\mathrm{NbN}$ [5]. Ils ont montré expérimentalement qu'il est possible d'obtenir une nette réduction de l'inductance cinétique sans dégradation notable des performances dynamiques de la jonction [6].

Dans ce qui suit, à l'aide d'une formulation analytique, nous déduisons toutes les caractéristiques magnétiques du système et en particulier la profondeur de pénétration équivalente du bilame et sun inductance totale. Nous comparons nos résultats à ceux issus du test [6] ainsi qu'à ceux issus de la résolution numérique utilisant une méthode énergétique variationnelle [7].

(*) Cette étude a été financée par le CEA-LETI Grenoble.

\section{Analyse théorique.}

La figure 1 représente la section transversale du dispositif étudié. Les rubans 1 et 2 (bilame) constituent l'électrode de base d'une jonction et le ruban 3, la contre-électrode. Les divers paramètres sont choisis en accord avec [6].

L'étude est faite en supposant la largeur $W$ des différents rubans très grande vis-à-vis des différentes profondeurs de pénétration $\lambda_{i}$. Dans ce cas, les effets de bord sont tout à fait négligeables; les équations de Maxwell montrent que le champ magnétique est suivant $x$ et le champ électrique possède une composante suivant $z$; ces grandeurs ne dépendent que de la position $y$ et s'écrivent de la façon suivante dans les différents milieux $i$ supraconducteurs (relations don-

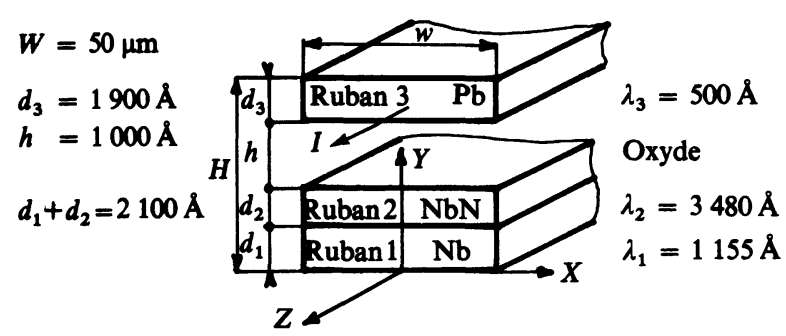

Fig. 1. - Section transversale du dispositif étudié.

[Cross section of the studied device.] 
nées dans le domaine fréquentiel) :

$$
\begin{aligned}
& H_{i}=A_{i} \exp \left(\frac{-y}{\lambda_{i}}\right)+B_{i} \exp \left(\frac{y}{\lambda_{i}}\right) \\
& E_{i}=j \omega \mu_{0} \lambda_{i}\left(A_{i} \exp \left(\frac{-y}{\lambda_{i}}\right)-B_{i} \exp \left(\frac{y}{\lambda_{i}}\right)\right) .
\end{aligned}
$$

En supposant le dispositif transportant un courant longitudinal $I$, la continuité des composantes $H_{x}$ et $E_{z}$ aux différentes interfaces ainsi que les conditions particulières :

$$
\begin{array}{ll}
H \equiv 0 & \text { pour } y \leqslant 0 \text { ou } y \geqslant H \\
H \equiv I / W & \text { pour } \quad d \leqslant y \leqslant d+h
\end{array}
$$

permettent de calculer les différents coefficients $A_{i}$ et $B_{i}$. Nous obtenons :

$$
\begin{aligned}
& A_{1}=\frac{\mathrm{I}}{W} \frac{\lambda_{2}}{D} \\
& B_{1}=-\frac{\mathrm{I}}{W} \frac{\lambda_{2}}{D} \\
& A_{2}=\frac{\mathrm{I}}{W} \times \\
& \times \frac{\left[\lambda_{1} \cosh \left(d_{1} / \lambda_{1}\right)-\lambda_{2} \sinh \left(d_{1} / \lambda_{1}\right)\right] \exp \left(d_{1} / \lambda_{2}\right)}{D} \\
& B_{2}=-\frac{\mathrm{I}}{W} \times \\
& \times \frac{\left[\lambda_{1} \cosh \left(d_{1} / \lambda_{1}\right)+\lambda_{2} \sinh \left(d_{1} / \lambda_{1}\right)\right] \exp \left(-d_{1} / \lambda_{2}\right)}{D}(8)
\end{aligned}
$$

avec :

$$
\begin{aligned}
D & =2\left[\lambda_{1} \cosh \frac{d_{1}}{\lambda_{1}} \sinh \frac{d_{2}}{\lambda_{2}}+\lambda_{2} \sinh \frac{d_{1}}{\lambda_{1}} \cosh \frac{d_{2}}{\lambda_{2}}\right] \\
A_{3} & =\frac{\mathrm{I}}{W} \frac{\exp \left[(d+h) / \lambda_{3}\right]}{1-\exp \left(-2 d_{3} / \lambda_{3}\right)} \\
B_{3} & =-\frac{\mathrm{I}}{W} \frac{\exp \left[-\left(d+h+2 d_{3}\right) / \lambda_{3}\right]}{1-\exp \left(-2 d_{3} / \lambda_{3}\right)}
\end{aligned}
$$

L'impédance de surface $Z_{\mathrm{s}}$ calculée en $y=d$ permet de définir la profondeur de pénétration équivalente pour le bilame à l'aide de la relation [8] :

$$
Z_{\mathrm{s}}=\left(\frac{E_{z}}{H_{x}}\right)_{y=d}=j \omega \mu_{0} \lambda_{\mathrm{eq}} .
$$

Compte tenu des valeurs des différentes constantes $A_{i}$ et $B_{i}$ données précédemment, nous obtenons :

$$
\lambda_{\text {eq }}=\frac{\lambda_{1 \text { eff }} \lambda_{2 \text { eff }}}{\lambda_{1 \text { eff }}+\lambda_{2 \text { eff }}}+\frac{\lambda_{2}^{2}}{\lambda_{1 \text { eff }}+\lambda_{2 \text { eff }}}
$$

avec

$$
\lambda_{i \text { eff }}=\lambda_{i} \operatorname{coth} \frac{d_{i}}{\lambda_{i}}
$$

La relation (12) montre clairement que la profondeur de pénétration équivalente du bilame se compose :

- d'un terme résultant de la mise en parallèle des rubans 1 et 2 ,

- d'un terme additionnel résultant du couplage existant entre ces rubans.

De plus l'énergie électromagnétique étant essentiellement confinée dans l'oxyde, le ruban 2 joue un rôle préférentiel vis-à-vis du ruban 1 . Pour des épaisseurs $d_{1}$ et $d_{2}$ données, on a donc intérêt à choisir un coefficient $\lambda_{2}$ inférieur à $\lambda_{1}$ si l'on veut minimiser la valeur de la pénétration équivalente $\lambda_{\text {eq }}$

La relation (11) appliquée en $y=d+h$ permet de définir la profondeur de pénétration effective du ruban 3. Nous retrouvons alors la relation (13) applicable au cas d'un seul ruban [9]. L'utilisation de la relation (12) dans le cas particulier où $\lambda_{1}=\lambda_{2}$ conduit aussi au résultat du ruban unique. Dans ces conditions, l'inductance totale équivalente au dispositif s'écrit :

$$
L=\frac{\mu_{0}}{W}\left(\lambda_{\text {eq }}+h+\lambda_{3 \mathrm{eff}}\right) .
$$

La relation de définition :

$$
\mathbf{B}=\operatorname{rot}(\mathbf{A})
$$

permet de calculer le potentiel vecteur A. Celui-ci est dirigé suivant $z$ et a pour expression :

$$
\mathcal{A}_{i}=\mu_{0} \lambda_{i}\left(A_{i} \exp \frac{-y}{\lambda_{i}}-B_{i} \exp \frac{y}{\lambda_{i}}\right)+\text { Cte. }
$$

Nous choisissons la constante arbitraire de façon à avoir un potentiel nul en $y=d$. Dans ce cas, l'équation de London :

$$
\mu_{0} \lambda^{2} \mathbf{J}+\mathbf{A}=\mathbf{0}
$$

conduit alors aux expressions suivantes pour les densités de courant :

$$
J_{i}=\left(\frac{A_{i}}{\lambda_{i}} \exp \left(-\frac{y}{\lambda_{i}}\right)-\frac{B_{i}}{\lambda_{i}} \exp \frac{y}{\lambda_{i}}\right)
$$

L'équation (17) associée aux conditions de continuité du champ magnétique et du potentiel vecteur entraîne les conditions suivantes à l'interface $\mathrm{Nb}-\mathrm{NbN}$ :

$$
\begin{aligned}
& \lambda_{1}^{2} \frac{\partial J_{1}}{\partial y}=\lambda_{2}^{2} \frac{\partial J_{2}}{\partial y}=H_{x} \\
& \lambda_{1}^{2} J_{1}=\lambda_{2}^{2} J_{2}=-\frac{\mathcal{A}_{z}}{\mu_{0}} .
\end{aligned}
$$


Ces relations montrent qu'il y a discontinuité de la densité de courant et de sa dérivée en $y=d_{1}$. En utilisant la relation (18) nous retrouvons ce résultat particulier.

3. Résultats. - En utilisant les paramètres géométriques et électriques donnés sur la figure 1 , nous avons calculé les variations de $\lambda_{\text {eq }}$ et de $L$ obtenues respectivement à partir des relations (12) et (14). Ces variations sont représentées sur la figure 2 en fonction de l'épaisseur $d_{1} \mathrm{du} \mathrm{Nb}$ pour une épaisseur totale $d=d_{1}+d_{2}$ constante égale à $2100 \AA$.

Le paramètre $\lambda_{\text {eq }}$ varie entre $\lambda_{2 \text { eff }}(6000 \AA)$ et $\lambda_{1 \text { eff }}(1200 \AA)$ lorsque $d_{1}$ passe de 0 à $d$. Pour de faibles épaisseurs de $\mathrm{Nb}$, nous observons des variations importantes de $\lambda_{\text {eq }}$ et $L$. Cette remarque peut être faite directement à partir de la relation (12).

Ces résultats théoriques comparés à ceux issus du test [6] et à ceux obtenus par résolution numérique [7] montrent la validité des relations analytiques proposées en (12) et (14).

En choisissant le courant traversant le ruban 3 tel que $I=1 \mathrm{~mA}$ et en fixant $d_{1}=900 \AA$ et $d_{2}=1200 \AA$, les différents coefficients peuvent être calculés à l'aide des équations (5) à (10). La figure 3 montre les variations de la densité de courant obtenues à partir de la relation (18). En particulier, à l'interface $\mathrm{NbN}-\mathrm{Nb}$, cette figure fait apparaître les discontinuités sur la densité de courant et sur sa dérivée qui vérifient les relations (19) et (20). Cette densité de courant n'est pas nulle sur la surface inférieure du ruban 1 ni sur la surface supérieure du ruban 3 .

Les variations du champ magnétique $\left(H_{x}\right)$ et du potentiel vecteur $\left(A_{z}\right)$ obtenues à partir des relations (1) et (16) sont représentées sur la figure 4 ; sur

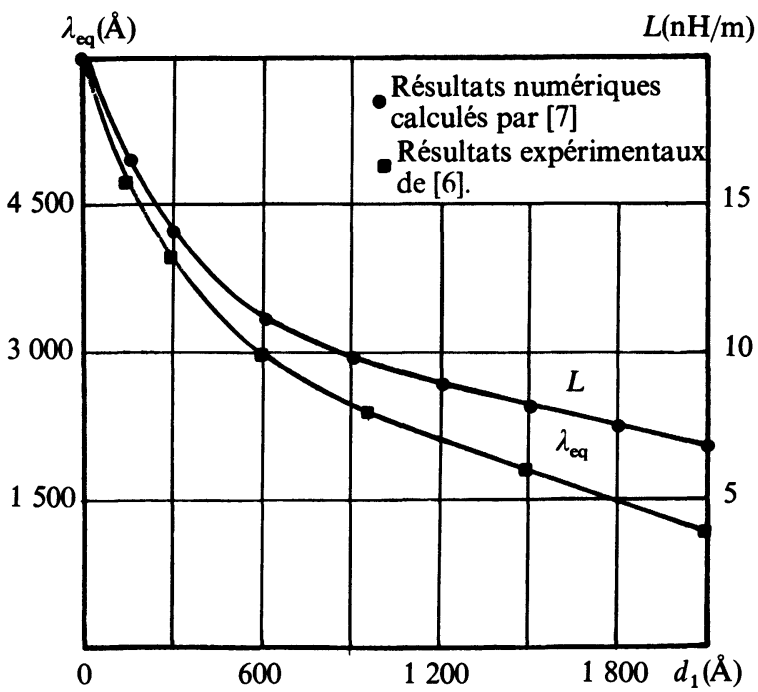

Fig. 2. - Variation de $\lambda_{\text {eq }}$ et de $L$ avec l'épaisseur $d_{1}$ du film $\mathrm{Nb}\left(d=d_{1}+d_{2}=2100 \AA\right)$.

[Variation of $\lambda_{\text {eq }}$ and $L$ with the $\mathrm{Nb}$ width film $\left(d=d_{1}+d_{2}=\right.$ $2100 \AA)$.]

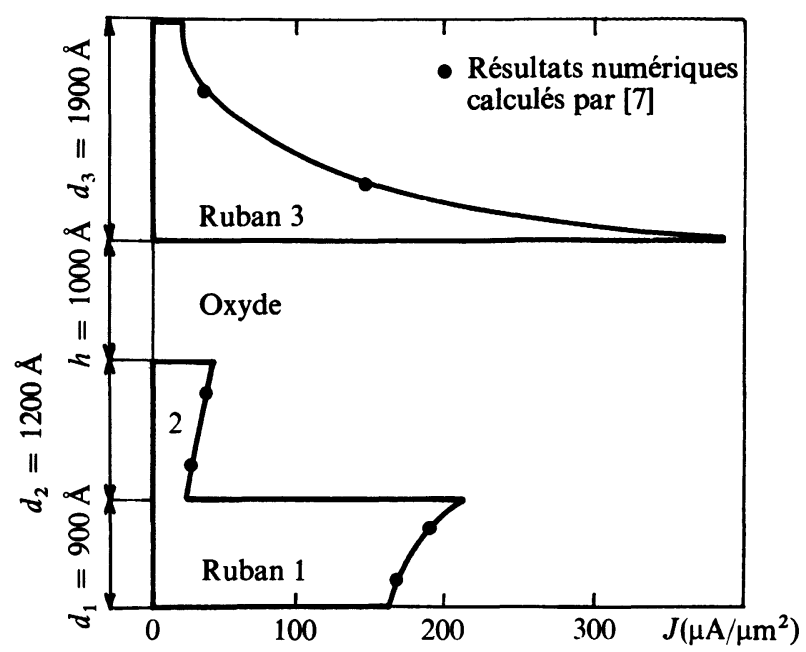

Fig. 3. - Distribution de $J$ en fonction de la position $y$ pour $I=1 \mathrm{~mA}$.

[Current density distribution $J$ with the $y$ position for $I=1 \mathrm{~mA}$.]

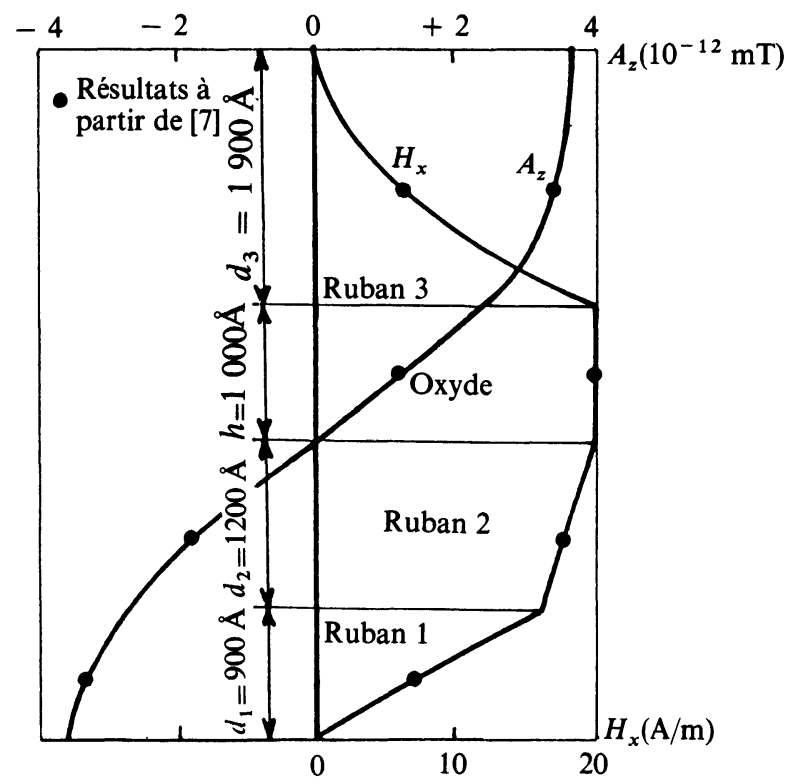

Fig. 4. - Variation de $H_{x}$ et $A_{z}$ en fonction de la position $y$ pour $I=1 \mathrm{~mA}$.

[Variation of $H_{x}$ and $A_{z}$ with the $y$ position for $I=1 \mathrm{~mA}$.]

l'interface NbN-Nb $\left(y=d_{1}\right)$ les valeurs de $H_{x}$ et $A_{z}$ vérifient bien les relations de discontinuités (19) et (20).

Sur les figures 3 et 4 nous avons porté les résultats obtenus par la méthode numérique décrite dans [7]. Le bon accord entre les deux approches justifie la validité de l'ensemble des relations analytiques proposées. 


\section{Conclusion.}

La méthode analytique que nous avons proposée nous a permis d'obtenir toutes les grandeurs magnétiques du dispositif supraconducteur étudié : profondeur de pénétration équivalente pour le bilame, inductance totale, distribution de la densité de courant, champ magnétique, potentiel vecteur.

La profondeur de pénétration équivalente $\lambda_{\text {eq }}$ fait apparaître un terme qui résulte de la mise en parallèle et un terme issu du couplage de deux rubans constituant le bilame. Le paramètre $\lambda_{\text {eq }}$ varie rapidement pour de faibles épaisseurs du ruban en niobium $\left(d_{1}\right)$. A l'interface des rubans 1 et 2 , les discontinuités de la densité de courant et de sa dérivée dépendent des profondeurs de London ainsi que du potentiel vecteur et du champ magnétique.

Cet effet de proximité total a été analysé dans le cas de rubans de largeur importante devant les différents paramètres $\lambda_{i}$ des divers matériaux utilisés. Pour des épaisseurs de $\mathrm{Nb}$ et $\mathrm{NbN}$ données, l'expression de $\lambda_{\text {eq }}$ montre que l'on a intérêt à choisir $\lambda_{2}$ plus faible que $\lambda_{1}$. Mais le choix de la nature des électrodes d'une jonction Josephson est imposé par d'autres considérations (technologiques, dynamiques...) et pratiquement, le critère précédent ne peut pas être respecté.

Dans le cas de rubans de faible largeur devant les profondeurs $\lambda_{i}$, les effets de bords ont un rôle non négligeable sur les distributions de densité de courant et seule une résolution numérique permet de traiter correctement le problème. Cependant, les remarques précédentes quant au choix des matériaux restent valables.

Dès que l'épaisseur $d_{1}$ devient comparable à $\lambda_{1}$, le ruban 2 est parcouru par un courant très faible, ce qui entraîne une inductance cinéque réduite.

L'utilisation de tels rubans bilames dans les liaisons de dispositifs logiques entraîne une réduction du temps de propagation et diminue l'importance des interconnexions dans la définition du temps de cycle du dispositif. Toute la modélisation proposée suppose le modèle de London justifié ; avec les matériaux utilisés et à la température de l'hélium liquide $(4 \mathrm{~K})$, cette hypothèse est valable jusqu'à $100 \mathrm{GHz}$ au moins. Les signaux véhiculés dans les interconnexions supraconductrices ayant des temps de montée de quelques picosecondes, leurs spectres sont très étendus mais ne vont guère au-delà de $100 \mathrm{GHz}$. Dans ce cas, les lignes peuvent être considérées comme non dissipatives et non dispersives : ce sont des lignes idéales qui n'apportent pas de déformation sur les signaux si elles sont adaptées.

L'ensemble de ces remarques, qui sont basées sur la réduction de l'inductance totale d'un bilame, doivent être prises en compte dans l'optimalisation des performances dynamiques des dispositifs interférométriques (analogiques ou logiques) à jonctions Josephson.

\section{Bibliographie}

[1] Kosaka, S., Shinoki, F., Takada, S., Hayakawa, H., IEEE Trans. Mag MAG-17 no 1 (1981) 314-317.

[2] Henkels, W. H., KiRCheR, C. J., IEEE Trans. Mag. MAG-13 no 1 (1977) 63-66.

[3] Matiso, J., I.B.M. J. 24 no 2 (1980) 113-129.

[4] Mason, P. V., Gould, R. W., J. Appl. Phys. 40 n 5 (1969) 2039-2051.

[5] Shoj,, A., Shinoki, F., Kosaka, S., Hayakawa, H., Jpn. J. Appl. Phys. 20 no $^{\circ}$ (1981) L587-L590.
[6] Shoj, A., Shinoki, F., Kosaka, S., Hayakawa, H., J. Appl. Phys. 32 no 3 (1981) 461-469.

[7] Chilo, J., Monllor, C., Rev. Phys. Appl. 17 (1982) 465-472.

[8] MaticK, R., Transmission lines for digital and communication networks (Mc Graw-Hill Book Co, N.Y.) 1969, Chap. VI.

[9] Swihart, J. C., J. Appl. Phys. 32 no 3 (1961) 461-469. 